\title{
Effects of Quadrat Size and Time of Year for Sampling of Verticillium dahliae and Lesion Nematodes in Potato Fields
}

T. A. Wheeler, Assistant Professor, Texas Agricultural Experiment Station, Rt. 3, Box 219, Lubbock 79401; L. V. Madden and R. C. Rowe, Professors, Department of Plant Pathology, The Ohio State University, Ohio Agricultural Research and Development Center, Wooster 44691; and R. M. Riedel, Professor, Department of Plant Pathology, The Ohio State University, Columbus 43210

\begin{abstract}
Wheeler, T. A., Madden, L. V., Rowe, R. C., and Riedel, R. M. 2000. Effects of quadrat size and time of year for sampling of Verticillium dahliae and lesion nematodes in potato fields. Plant Dis. 84:961-966.

Six potato fields were randomly sampled using quadrat areas ranging from $10 \mathrm{~m}^{2}$ to $4,000 \mathrm{~m}^{2}$. A composite soil sample consisting of 20 soil cores was taken from each of 20 quadrats of each unit area. All samples were assayed for Verticillium dahliae and nematodes. The area in which a composite sample was taken had little effect on estimated mean and variance of $V$. dahliae until quadrat size was $\geq 1,000 \mathrm{~m}^{2}$. Then variances dramatically increased with size. With Pratylenchus spp., there was no systematic relationship between quadrat size and variance. Seven additional fields were divided into 2,000 or $4,000 \mathrm{~m}^{2}$ grids, and each grid was sampled in both spring and fall for $V$. dahliae and plant-parasitic nematodes (Pratylenchus spp. and Meloidogyne hapla). Density of $V$. dahliae was lower, and in some cases, degree of aggregation was higher, in the spring than in the fall. Sampling for $V$. dahliae in the fall required 2 to 7 composite samples to estimate mean density within $25 \%$ of the true mean, while in the spring, 10 to 19 samples were required. Assays of Pratylenchus spp. required a similar number of samples in spring and fall surveys, while $M$. hapla required fewer samples with spring than with fall sampling when estimating densities $>20$ second-stage juveniles per $100 \mathrm{~cm}^{3}$ of soil.
\end{abstract}

Verticillium dahliae Kleb. can cause premature senescence and lower yields in potato, cotton, and many other crops $(7,18,19)$. When the lesion nematode, Pratylenchus penetrans (Cobb) Filipjev \& Schuur., and $V$. dahliae are both present, plant disease symptoms (senescence) and yield losses are more severe than with $V$. dahliae alone $(12,14)$. Root-knot nematode (Meloidogyne hapla Chitwood) may be involved in similar disease complexes $(10,28)$. Microsclerotia in soil are the primary infective propagules of $V$. dahliae during early crop development $(19,22)$. These propagules can be recovered from soil and enumerated with semiselective media (16). Considerable efforts have been made to devise sampling procedures for microsclerotia to reflect more accurately the preplant propagule density for use in determining appropriate management strategies $(16,28,30)$.

To maximize benefits from sampling soil, estimates of mean and variance of pathogen density (and hence aggregation) are important. Various measurements of aggregation or heterogeneity have been applied to sampling data for $V$. dahliae

Corresponding author: T. A. Wheeler

E-mail: ta-wheeler@tamu.edu

Accepted for publication 26 May 2000.

Publication no. D-2000-0620-02R

(C) 2000 The American Phytopathological Society
$(5,11,25,28)$. In general, propagules of $V$. dahliae have been found to be aggregated in soil and often can be described by the negative binomial distribution. Thus, many samples are required to achieve a coefficient of variation $(c v)$ of less than 10 to $25 \%$ (28). The area over which a composite soil sample is taken may affect the estimate of mean density for an organism. In previous studies, population density of $V$. dahliae was measured from a single-sized, consecutive quadrat in fields; then density determinations for single quadrats were combined (mathematically, not experimentally) to estimate larger quadrat areas (28). The objectives of this study were: (i) to test the influence of quadrat size on estimates of mean and variance, and hence aggregation, of $V$. dahliae and Pratylenchus spp. in commercial potato fields; and (ii) to compare fall versus spring sampling for aggregation of $V$. dahliae, Pratylenchus spp., and M. hapla.

The information we gained was then combined with information from other sampling studies $(3,5,11,16,25,28,30)$ to define a sampling plan. The sampling plan was combined with a curvilinear yield-loss model (29) to define the number of samples necessary to predict yield loss and a subsequent management strategy.

\section{MATERIALS AND METHODS}

Quadrat size. Six commercial potato fields in Ohio were sampled repeatedly to determine the effects of quadrat size on estimated mean and variance for soil populations of $V$. dahliae and Pratylenchus spp. Field 1 (14 ha) was a silt-loam soil that had been in a potato-wheat rotation for at least 50 years. Field 2 ( 5 ha) was a silt-loam soil that had been in a potatowheat rotation for at least 12 years. Fields 3 to 6 were coarse-textured soils that had been in potato-grain crop rotations for $<10$ years. Fields 3 (24 ha), 4 (7 ha), 5 (19 ha), and 6 (15 ha) were located in northwestern Ohio. Fields 1 and 2 were located in northeastern Ohio. Each of these six fields was divided into contiguous quadrats of 10 , $50,100,250$, and $1,000 \mathrm{~m}^{2}$, and fields 1 , 3,5 , and 6 (because of their larger size) were also divided into quadrats of 4,000 $\mathrm{m}^{2}$. The quadrats were all assigned consecutive identifying numbers; for example, in a 15-ha field there would be 15,000 consecutive numbers for the $10 \mathrm{~m}^{2}$ quadrat area, 3,000 for the $50 \mathrm{~m}^{2}$ quadrat area, 1,500 for the $100 \mathrm{~m}^{2}$ quadrat area, etc. Twenty randomly selected quadrats of each size were sampled for each of the six fields. The position of each quadrat was determined by using a random number generator from the procedure PLAN in SAS (SAS Institute, Cary, NC). A seed number is used in this procedure to generate each set of random numbers, and the seed numbers were selected without pattern. The locations of the selected areas (100 to 120 per field, depending on whether there were $4,000 \mathrm{~m}^{2}$ quadrats) were marked with flags, and 20 soil cores per quadrat $(2.5 \mathrm{~cm}$ diameter, $20 \mathrm{~cm}$ deep) were taken using a zigzag pattern that covered the entire area of the quadrat. Cores from each quadrat were mixed in a bucket, and a subsample of $1,000 \mathrm{~cm}^{3}$ was removed and placed in a plastic bag for assays. All samples from a field were collected over a 1- to 2-day period.

To assay for $V$. dahliae, soil samples were dried for 2 weeks at room temperature $\left(\sim 25^{\circ} \mathrm{C}\right)$, and then each sample was smoothed with a rolling pin, bagged, and kept at $10^{\circ} \mathrm{C}$ until tested $(<6$ months). For each sample, a dilution plate assay was conducted twice. This consisted of taking $20 \mathrm{~cm} 3$ of soil from each sample, placing in $80 \mathrm{ml}$ of deionized water, stirring for approximately 30 $\mathrm{s}$, and then plating five 1-ml aliquots on semiselective sodium polypectate media 
(16) in sterilized glass petri plates. After incubating plates in the dark for 13 days at $25^{\circ} \mathrm{C}$, soil on plates was removed by washing, and $V$. dahliae colonies in the agar were identified and counted under a dissecting microscope.

To assay for Pratylenchus spp., $200 \mathrm{~cm}^{3}$ of soil from each sample was placed in a pie-pan apparatus ( $<1$ week after sampling), and nematodes were collected after 36 to 48 h (27). Plant-parasitic nematodes were identified and counted, and samples containing lesion nematodes were treated with an equal volume of boiling water, concentrated to 5 to $10 \mathrm{ml}$ with a sieve of 25- $\mu \mathrm{m}$ pore size, and then treated with an equal volume of $10 \%$ formaldehyde. Representative adults were mounted on microscope slides, and species were determined (8).

Analyses. For each set of quadrat sizes, the mean and variance among the 20 quadrats of that size were determined for $V$. dahliae and Pratylenchus spp., and homogeneity of variances was compared among quadrat areas by two tests, Box's and Levene's $(P=0.05)(15)$. Both tests were used because little is known about how these tests may differ in determining how population variance of potato early dying pathogens may be similar or different. In addition, a statistical model was developed by using an expansion of Taylor's power law $(3,26)$ for all fields and quadrat sizes. The full model was

$$
\begin{aligned}
\log (v)_{i j}= & \log (a)+b \log (m)+F_{i}+ \\
& b_{i} \log (m)+S_{j}+b_{j} \log (m)+\text { error }
\end{aligned}
$$

in which $v$ is the variance, $m$ is the mean density, $\log (a)$ is the intercept (predicted $\log (v)$ when $\log (m)=0), b$ is a slope, $F_{i}$ is a factor for field $(i=1, \ldots, 6), S_{j}$ is a factor for quadrat size $(j=1, \ldots 6)$, and $b_{i}$ and $b_{j}$ are slope components of $\log (v)$ in relation to $\log (m)$ for the six quadrat sizes and six fields, respectively. The factors $F_{i}$ and $S_{j}$ are grouping or class variables, not continuous variables, with $5 \mathrm{df}$ each. There are also $5 \mathrm{df}$ for both $b_{i}$ and $b_{j}$, because there are six levels of quadrat size and field. The error term in the equation is considered to be equivalent to the sum of $F_{i} S_{j}$ and threeway interaction term. If quadrat size and field had no effect on the magnitude of $v$ or the rate at which $v$ increased with $m$, then equation 1 reduces to

$\log (v)=\log (a)+b \log (m)+$ error

which is the standard power law $(3,26)$. The reason for using equation 1 is that a linear relationship between $\log (v)$ and $\log (m)$ is expected for density of many organisms $(3,26)$, including $V$. dahliae (28), and equation 1 allows testing for additional sources of variation in $\log (v)$ after correcting for $\log (m)$. Significance of the terms in equation 1 was tested with reduced versus full model $F$ tests.

Time of sampling. A comparison was made between soil samples collected in the fall versus spring for accuracy of pathogen density estimates. Seven fields, different from the six fields in the previous study, were selected. Four of the fields were of a coarse-textured soil located in northwest Ohio, and three fields were finer-textured (silt loam) soils located in northeastern Ohio. Soil samples were collected in the spring (April to May) and fall (September to October). Field sizes ranged from 2.4 to 14.4 ha. Each field was divided entirely into 4,000 or $2,000 \mathrm{~m}^{2}$ quadrats (depending on the size of the field), and 20 soil cores were collected from each of the quadrats in a zigzag pattern, mixed, and a $1,000 \mathrm{~cm}^{3}$ subsample was assayed for $V$. dahliae and plant-parasitic nematodes. Assay procedures were as described previously. The mean and variance of $V$. dahliae, Pratylenchus spp., and Meloidogyne populations from each field were used in regression analysis with an expanded power law similar to equation 1 , but with the factor $T_{j}$ $(j=1,2)$ for season (time) instead of $S_{j}$. The number of samples required to estimate the population density of each organism within $25 \%$ of the true mean (i.e., with a $c v$ of 0.25 ) was calculated over the density range of 10 to 100 (units depend on

Table 1. Estimated mean density, variance (VAR), and aggregation index $(k)$ of Verticillium dahliae microsclerotia from sampling a range of quadrat unit

\begin{tabular}{|c|c|c|c|c|c|c|c|c|c|c|c|c|c|c|c|c|c|c|}
\hline \multirow[b]{3}{*}{ Field } & \multicolumn{18}{|c|}{ Quadrat sampling size $\left(\mathbf{m}^{2}\right)$} \\
\hline & \multicolumn{3}{|c|}{10} & \multicolumn{3}{|c|}{50} & \multicolumn{3}{|c|}{100} & \multicolumn{3}{|c|}{250} & \multicolumn{3}{|c|}{1,000} & \multicolumn{3}{|c|}{4,000} \\
\hline & Mean & VAR & $k^{\mathbf{a}}$ & Mean & VAR & $k$ & Mean & VAR & $k$ & Mean & VAR & $k$ & Mean & VAR & $k$ & Mean & VAR & $\boldsymbol{k}$ \\
\hline 1 & 6.4 & 18 & 3.5 & 3.6 & 10 & 2.0 & 77 & 38 & 1.9 & 7.0 & 25 & 2.7 & 5.9 & 24 & 1.9 & 7.2 & 13 & 9.5 \\
\hline 2 & 1.9 & 5 & 1.1 & 1.5 & 5 & 0.5 & 1.6 & 3 & 2.2 & 1.8 & 4 & 1.7 & 2.3 & 4 & 2.8 & $\ldots$ & $\ldots$ & \\
\hline $3^{\mathrm{b}}$ & 8.3 & 99 & 0.7 & 5.0 & 38 & 0.7 & 4.8 & 43 & 0.5 & 6.9 & 37 & 1.5 & 35.9 & $1 \times 10^{4}$ & 0.0 & 12.2 & 55 & 3.4 \\
\hline 4 & 9.8 & 109 & 0.9 & 12.5 & 230 & 0.7 & 11.8 & 236 & 0.6 & 11.5 & 99 & 1.5 & 11.7 & 117 & 1.2 & & & \\
\hline $5^{\mathrm{b}}$ & 6.0 & 147 & 0.2 & 14.1 & 588 & 0.3 & 8.4 & 99 & 0.7 & 2.6 & 15 & 0.5 & 5.8 & 39 & 1.0 & 120.6 & $2 \times 10^{5}$ & 0.0 \\
\hline 6 & 7.0 & 36 & 1.6 & 10.8 & 160 & 0.7 & 7.2 & 46 & 1.3 & 10.3 & 80 & 1.5 & 12.6 & 149 & 1.1 & 11.5 & 59 & 2.7 \\
\hline
\end{tabular}
sizes in commercial potato fields in Ohio

${ }^{a}$ The aggregation parameter $k$ was calculated as $k=\left[\left(\right.\right.$ mean $\left.^{2}-\mathrm{VAR} / n\right) /(\mathrm{VAR}-$ mean $\left.)\right]$, where $n$ was the number of samples collected for a sampling unit size, $n=20$.

b These fields had significant differences among variances in quadrat areas according to Levene's test, but variances were homogeneous according to Box's test (15).

Table 2. Estimated mean density, variance (VAR), and aggregation index ( $k$ ) of Pratylenchus spp. ${ }^{a}$ vermiforms from sampling a range of quadrat sizes in

\begin{tabular}{|c|c|c|c|c|c|c|c|c|c|c|c|c|c|c|c|c|c|c|}
\hline \multirow[b]{3}{*}{ Field } & \multicolumn{18}{|c|}{ Quadrat sampling size $\left(\mathbf{m}^{2}\right)$} \\
\hline & \multicolumn{3}{|c|}{10} & \multicolumn{3}{|c|}{50} & \multicolumn{3}{|c|}{100} & \multicolumn{3}{|c|}{250} & \multicolumn{3}{|c|}{1,000} & \multicolumn{3}{|c|}{4,000} \\
\hline & Mean & VAR & $k^{\mathbf{b}}$ & Mean & VAR & $k$ & Mean & VAR & $k$ & Mean & VAR & $k$ & Mean & VAR & $k$ & Mean & VAR & $k$ \\
\hline $1^{\mathrm{c}}$ & 40.2 & 1,165 & 1.4 & 37.2 & 1,012 & 1.4 & 26.1 & 462 & 1.5 & 31.1 & 435 & 2.3 & 39.3 & 1,167 & 1.3 & 21.8 & 491 & 1.0 \\
\hline $2^{\mathrm{c}, \mathrm{d}}$ & 50.0 & 858 & 3.0 & 83.3 & 3,255 & 2.1 & 74.2 & 1,450 & 3.9 & 63.9 & 1,169 & 3.6 & 78.1 & 1,453 & 4.3 & & & \\
\hline $3^{c}$ & 96.5 & 17,487 & 0.5 & 46.0 & 2,836 & 0.7 & 19.5 & 921 & 0.4 & 53.0 & 7,632 & 0.3 & 67.2 & 4,151 & 1.1 & 52.6 & 3,454 & 0.8 \\
\hline $4^{c}$ & 11.5 & 371 & 0.3 & 22.0 & 1,427 & 0.3 & 8.5 & 203 & 0.3 & 17.0 & 759 & 0.3 & 10.5 & 152 & 0.7 & & & \\
\hline 5 & 8.5 & 119 & 0.6 & 6.8 & 212 & 0.2 & 13.2 & 189 & 0.9 & 14.5 & 342 & 0.6 & 14.7 & 249 & 0.9 & 13.9 & 237 & 0.8 \\
\hline 6 & 7.5 & 136 & 0.4 & 7.0 & 212 & 0.2 & 9.5 & 216 & 0.4 & 15.8 & 648 & 0.3 & 14.7 & 293 & 0.7 & 6.5 & 66 & 0.7 \\
\hline
\end{tabular}
commercial potato fields in Ohio

${ }^{a}$ Mean percent Pratylenchus penetrans ranges from 1 to $10 \%$ in field 1, 1 to 5\% in field 2, 26 to $48 \%$ in field 3, 53 to $97 \%$ in field 4, 54 to $78 \%$ in field 5, and 60 to $100 \%$ in field 6 .

b The aggregation parameter $k$ was calculated as $k=\left[\left(\operatorname{mean}^{2}-(\mathrm{VAR} / n)\right] /(\mathrm{VAR}-\right.$ mean $)$, where $n$ was the number of samples taken in a sampling unit area.

$c$ Variances were significantly different among quadrat areas in these fields according to Levene's test (15).

${ }^{d}$ Variances were significantly different among quadrat areas in this field according to Box's test (15). 
organism) by the equation 13.7 given in Campbell and Madden (3).

\section{RESULTS}

Quadrat size. The estimated mean density of $V$. dahliae in commercial potato fields was not affected by the different and field $5\left(4,000 \mathrm{~m}^{2}\right)$ (Table 1$)$. The estimated mean density of the population of Pratylenchus spp. (which consisted of a mixture of $P$. penetrans, $P$. crenatus, and $P$. scribneri) differed widely among quadrat sizes in a field, but there was no trend of higher mean or variance associated with any particular quadrat size (Table 2). The two variance-testing methods gave somewhat different results. With Box's test, homogeneity of variances for $V$. dahliae was found in all fields, while for Pratylenchus spp., in all but one field variances were homogeneous across the different quadrat sizes (Tables 1 and 2). However, with Levene's test, heterogeneity of variances between quadrat sizes for $V$. dahliae was found in two of six fields (Table 1) and for Pratylenchus spp. in four of six fields (Table 2). Large quadrat sizes (1,000 or $4,000 \mathrm{~m}^{2}$ ) were associated with larger variances for $V$. dahliae in two fields (fields 3 and 5). In both cases, a single sample was responsible for the higher than normal mean and variance within the 20 samples assayed (data not presented). With Pratylenchus spp., there was no apparent relationship between variance and quadrat size.

In fitting the expanded power model (equation 1) to the data for $V$. dahliae, the quadrat sizes except for field $3\left(1,000 \mathrm{~m}^{2}\right)$

$\log (m)$ term was highly significant $(P<$ $0.01)$. The factor $b_{i} \log (m)$ (interaction of field size $\left[F_{i}\right]$ and mean density) was not significant $(P>0.20)$, and $F_{i}$ was borderline $(P=0.045)$. The other terms were highly significant $(P<0.01)$, and thus the reduced model can be written as

$$
\begin{aligned}
\log (v)_{j}= & \log (a)+b \log (m)+S_{j} \\
& +b_{j} \log (m)+\text { error }
\end{aligned}
$$

which means that the intercept $\left[\log (a)+S_{j}\right]$ and slope $\left(b+b_{j}\right)$ from the reduced model for the relationship between $\log (v)$ and $\log (m)$ were dependent on quadrat size. The slopes and intercepts for each quadrat size (but across all fields), were obtained from separate regressions (Table 3). These estimated parameters were compared with $t$ tests $(P=0.05)$, which showed that the largest quadrat size $\left(4,000 \mathrm{~m}^{2}\right)$ had a significantly higher slope and lower intercept than all but the next largest size $(1,000$ $\mathrm{m}^{2}$ ). The smaller quadrat sizes had similar slopes and intercepts and were not different from each other. The general trend was for increasing slope and decreasing intercept of the relation between $\log (v)$ and $\log (m)$ with increasing quadrat size, starting at 250 $\mathrm{m}^{2}$. The significant effect of $F_{i}$ (not shown in equation 3 ) indicates that the intercepts (but not slopes) can vary with field (intercept equal to $\log (a)+S_{j}+F_{i}$ ). The intercept for any given field can be obtained by adding the following value to the appropriate quadrat-size intercept (Table 3): 0 for field $1,0.48$ for field $2,0.27$ for field 3, 0.09 for field $4,0.48$ for field 5, and -0.04 for field 6 .

Table 3. The effect of quadrat size on variance-mean relations when applying Taylor's power law ${ }^{\mathrm{a}}$ to population estimates for Verticillium dahliae

\begin{tabular}{ccccc}
\hline $\begin{array}{l}\text { Quadrat size } \\
\left(\mathbf{m}^{2}\right)\end{array}$ & Intercept $\left(\mathbf{S E}^{\mathbf{b}}\right)$ & Slope (SE) & $\begin{array}{c}\text { Coefficient of } \\
\text { determination }\end{array}$ & Prob. $>\boldsymbol{t}$ \\
\hline 10 & $0.52(1.13)$ & $1.81(0.62)$ & 0.68 & 0.04 \\
50 & $0.47(0.52)$ & $2.01(0.26)$ & 0.94 & 0.01 \\
100 & $0.05(0.53)$ & $2.06(0.28)$ & 0.93 & 0.01 \\
250 & $0.74(0.40)$ & $1.51(0.22)$ & 0.92 & 0.01 \\
1,000 & $-1.48(0.86)$ & $2.83(0.37)$ & 0.94 & 0.01 \\
4,000 & $-4.54(0.29)$ & $3.51(0.09)$ & 1.00 & 0.01 \\
\hline
\end{tabular}

a Taylors power law is: $\log ($ variance $)=\log (a)+b \log ($ mean $)$, in which $\log (a)$ and $b$ are the intercept and slope, respectively (26).

${ }^{\mathrm{b}} \mathrm{SE}$ is the standard error.
For the lesion nematodes, neither quadrat size nor any interaction had significant effects $(P>0.20)$. However, $\log (m)$ was highly significant $(P<0.01)$, giving the equation

$$
\begin{aligned}
\log (v)= & 1.02(\mathrm{SE}=0.176)+ \\
& 1.31(\mathrm{SE}=0.125) \log (m)
\end{aligned}
$$

which had an $R^{2}$ of 0.78 . The field parameter was significant $(P=0.04)$, indicating that the intercept depended on field. The intercept for a given field is obtained by adding the following number to 1.02 of equation 4: 0 for field $1,-0.22$ for field 2 , 0.37 for field $3,0.37$ for field $4,0.17$ for field 5, and 0.27 for field 6 .

Time of sampling. Population means and variances for $V$. dahliae, Pratylenchus spp., and M. hapla populations were estimated for soil samples taken in the spring and the fall (Table 4). Estimated mean density values for $V$. dahliae from fall samples ranged from 2 to 79 microsclerotia per $\mathrm{cm}^{3}$ soil, and from spring samples ranged from 1 to 31 microsclerotia per $\mathrm{cm}^{3}$ soil. In all cases, the mean densities assayed for each field were lower in the spring than in the fall (Table 4). Variances increased with increasing density of $V$. dahliae. The aggregation index, $k$, was lower in spring than in fall samples (i.e., increased aggregation) in three (fields b, c, f) of seven cases (Table 4). In three cases (fields a, d, h), mean equaled the variance in spring samples, leading to a decrease in aggregation (because $k$ is equal to infinity when $m=v$ ) compared with fall sampling (Table 4). In field g, aggregation was similar between spring and fall sampling, although both mean and variance were smaller in the spring than in the fall (Table 4).

For Pratylenchus spp., estimated mean density ranged from 53 to 107 vermiforms per $100 \mathrm{~cm}^{3}$ of soil for fall samples and 40 to 220 vermiforms per $100 \mathrm{~cm}^{3}$ of soil for spring samples (Table 4). Variances for Pratylenchus spp. based on spring and fall samples were much greater than the estimated mean densities, indicating a high degree of aggregation. $M$. hapla estimated mean densities ranged from 0 to $183 \mathrm{sec}-$ ond-stage juveniles per $100 \mathrm{~cm}^{3}$ of soil for

\begin{tabular}{|c|c|c|c|c|c|c|c|c|c|c|c|c|c|c|}
\hline \multirow[b]{3}{*}{ Field } & \multicolumn{6}{|c|}{ V. dahliae } & \multicolumn{4}{|c|}{ Pratylenchus spp. } & \multicolumn{4}{|c|}{ M. hapla } \\
\hline & \multicolumn{3}{|c|}{ Fall } & \multicolumn{3}{|c|}{ Spring } & \multicolumn{2}{|c|}{ Fall } & \multicolumn{2}{|c|}{ Spring } & \multicolumn{2}{|c|}{ Fall } & \multicolumn{2}{|c|}{ Spring } \\
\hline & Mean & VAR & $k^{\mathbf{a}}$ & Mean & VAR & $k$ & Mean & VAR & Mean & VAR & Mean & VAR & Mean & VAR \\
\hline a & 17 & 110 & 3.1 & 3.1 & 3.3 & 48 & 71 & 1,962 & 40 & 930 & 14 & 449 & 18 & 1,176 \\
\hline $\mathrm{b}$ & 79 & 3,170 & 2.0 & 31 & 1,780 & 0.5 & 103 & 10,384 & 66 & 4,369 & 22 & 1,954 & 1 & 16 \\
\hline $\mathrm{c}$ & 28 & 225 & 3.9 & 8 & 48 & 1.5 & 68 & 2,016 & 41 & 900 & 183 & 68,854 & 7 & 408 \\
\hline d & 37 & 104 & 20 & 5 & 5 & $\ldots{ }^{b}$ & 107 & 3,588 & 220 & 7,430 & 0 & 1 & 5 & 40 \\
\hline f & 41 & 132 & 18 & 13 & 74 & 2.8 & 76 & 1,632 & 166 & 18,333 & 13 & 292 & 11 & 286 \\
\hline $\mathrm{g}$ & 22 & 172 & 3.2 & 7 & 20 & 3.5 & 53 & 2,421 & 150 & 4,449 & 3 & 36 & 10 & 159 \\
\hline $\mathrm{h}$ & 2 & 3 & 4.0 & 1 & 1 & $\ldots^{\mathrm{b}}$ & 98 & 3,329 & 56 & 3,080 & 10 & 94 & 15 & 734 \\
\hline
\end{tabular}
fall samples, and 1 to 18 second-stage ju-

Table 4. Estimated means and variance (VAR) for Verticillium dahliae, Pratylenchus spp., and Meloidogyne hapla populations for fall and spring samples collected in seven commercial potato fields in Ohio

a $k$ is an aggregation index estimated by the formula: mean $2 /(\mathrm{VAR}-$ mean).

${ }^{\mathrm{b}}$ No aggregation index could be calculated because the mean $=$ variance. 
veniles per $100 \mathrm{~cm}^{3}$ of soil for spring samples. Variances for $M$. hapla were also greater than estimated mean densities for both spring and fall samples (Table 4).
With the expanded power law model for the seasonal data, $\log (m)$ was highly significant $(P<0.01)$, season and the interaction of season and $\log (m)$ were significant
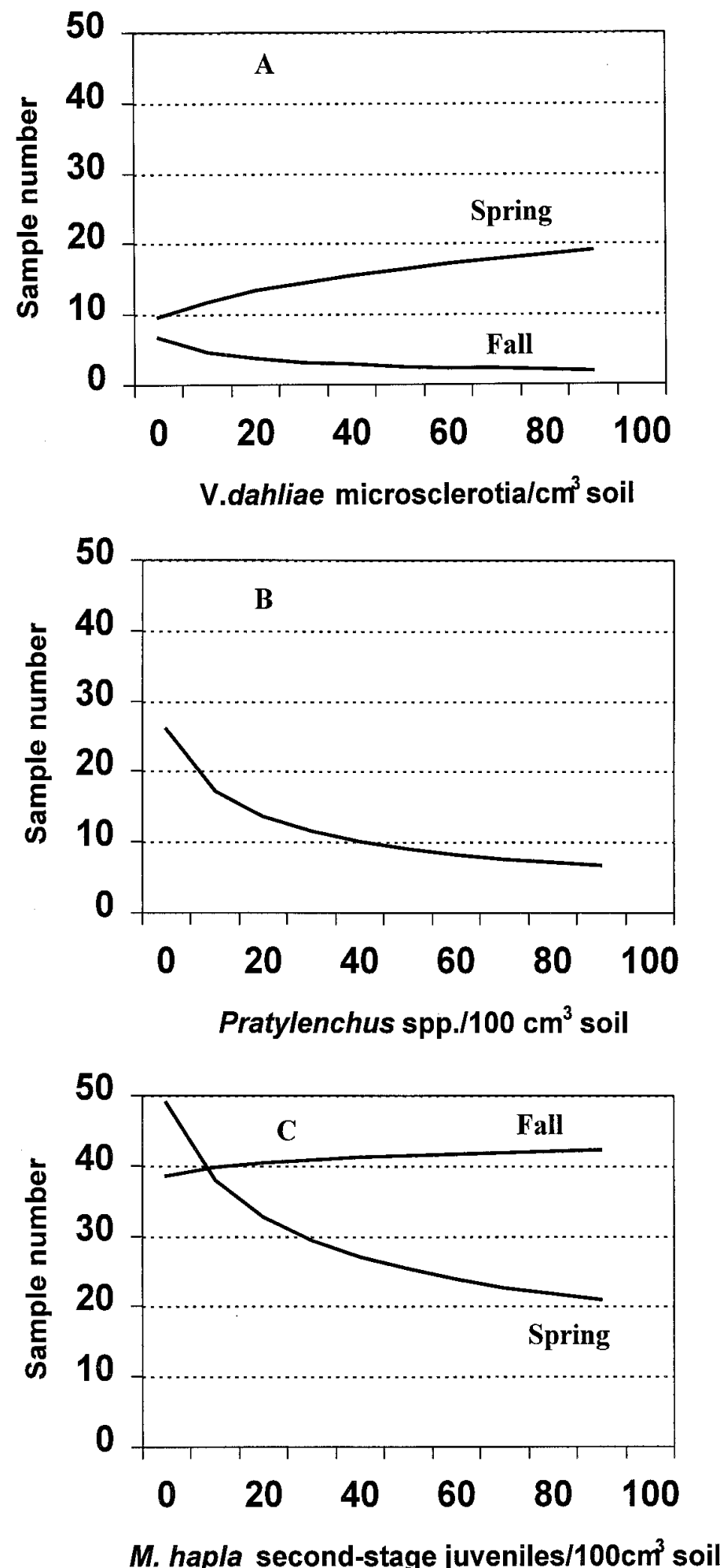

Fig. 1. Number of samples required to estimate population densities of Verticillium dahliae (A), Pratylenchus spp. (B), and Meloidogyne hapla $(\mathbf{C})$ during fall and spring sampling within $25 \%$ of the true mean. The formula for calculating sampling number was: $n=a\left(\right.$ mean $\left.^{(b-2)}\right) / C^{2}$, where $n$ is the sampling number, $a$ and $b$ were parameters estimated from Taylor's power law (26), and $C$ is the coefficient of variation set arbitrarily at 0.25 . Estimated $a$ and $b$ parameters for lines are: (A) spring $a$ $=0.30, b=2.30$; fall $a=1.47, b=1.46$; (B) $a=6.30, b=1.41$; (C) spring $a=7.17, b=1.63$; fall $a=$ $2.20, b=2.04$. There was no difference in sampling parameters between spring and fall for Pratylenchus spp., so only one line is presented.

for $V$. dahliae and M. hapla $(P<0.05)$, but not for Pratylenchus spp. $(P>0.20)$. All other terms were not significant $(P>0.10)$. Estimated parameters are given in the legend of Figure 1.

Sample size. For $V$. dahliae, greater sampling intensity was required in the spring than in the fall (Fig. 1A) because of the lower mean density and in some cases higher aggregation in the spring. Quadrat size was kept constant during both spring and fall sampling, so that assessed aggregation was a function of heterogeneity of the organisms and not of quadrat size. Sampling intensity for $V$. dahliae also increased as mean density increased for spring sampling due to power law $b>2$ (Fig. 1A). In the fall, sampling intensity decreased as mean density increased (Fig. 1A). Two to seven samples were adequate to achieve $c v=0.25$ for fall sampling for $V$. dahliae over a range of tested fungal densities, while for spring sampling, sample number ranged from 9 to 19 to achieve the same $c v$. Moreover, at low densities of $V$. dahliae ( 10 microsclerotia per $\mathrm{cm}^{3}$ of soil), sampling intensity was similar for both fall and spring sampling (Fig. 1A).

For Pratylenchus spp., there was no difference between predicted spring and fall sampling numbers because there was no difference in power-law parameters. Sampling intensity declined as mean density increased because $b<2$ (Fig. 1B). For $M$. hapla, fewer samples were necessary in the spring than in the fall when density of second-stage juveniles was $>20$ per $100 \mathrm{~cm}^{3}$ of soil (Fig. 1C). However, more than 20 samples were necessary for all M. hapla densities calculated.

\section{DISCUSSION}

Risk of yield losses due to potato early dying is a function of preplant population density of $V$. dahliae, the presence or absence of $P$. penetrans, susceptibility of host cultivar, and weather conditions $(18,20,28)$. Severity and incidence of plants with potato early dying increases with density of $V$. dahliae $(12,14,29)$. Yield loss has been approximated (27) with the curvilinear equation $y(V D)=b_{0}+(1-$ $\left.b_{0}\right) /[1+(V D / 36.7)]$, where $b_{0}$ represents the minimum yield (or 1 - maximum yield loss due to the disease) and $V D$ is the density of $V$. dahliae. The minimum yield $\left(b_{0}\right)$ is lowered by the presence of a threshold density of $P$. penetrans (29) (from 0.55 to 0.23 under homogeneous conditions) and is also affected by environment. The severity of potato early dying symptoms is not directly related to $P$. penetrans density when density is $\geq 80$ vermiforms per $100 \mathrm{~cm}^{3}$ of soil (21). The mechanism of the interaction between $V$. dahliae and $P$. penetrans has not been determined $(2,7)$. Under conditions favorable for disease (cool and wet early in the season), the minimum yield is lower and disease severity is higher, while under unfavorable disease conditions (hot 
and dry), minimum yield is higher and disease severity is lower (4). The isolate of $V$. dahliae can also modify estimates of $b_{0}$, so that a more aggressive isolate would have a lower minimum yield than a less aggressive isolate (1).

The yield loss equation above was generated from homogeneous populations of $V$. dahliae and $P$. penetrans. In a heterogeneous environment, yield loss equations should be modified to account for spatial aggregation on the nonlinear response of yield to $V D(6,9,13,17,23)$. One approach is based on use of an expansion of the yield model to obtain mean yield, $\bar{y}(V D)$ :

$$
\bar{y}(V D)=y(\overline{V D})+y^{\prime \prime}(\overline{V D}) v(V D) / 2
$$

in which $v(V D)$ is the variance of $V D$ in the field, $\overline{V D}$ is mean $V D$ in the field, and $y^{\prime \prime}(\overline{V D})$ is the second derivative of $y(\overline{V D})$ evaluated at $\overline{V D}$, which equals $2(1-$ $\left.b_{0}\right) /[1,346.89(1+\overline{V D} / 36.7)]^{3}$. Thus, predicted mean yield is dependent on variability of $V D$, which is a direct function of aggregation of $V D$. Aggregation may be a function of the number of years a field has been in a potato rotation, fumigation history, spatial heterogeneity of soil factors such as organic matter, and other factors. A heterogeneous field, in terms of $V$. dahliae and $P$. penetrans densities, results in greater mean yield and hence less loss from disease than a homogeneous case with the same average density of $V$. dahliae (Table 5).

Aggregation of the pathogen plays a second role in assessing risk of early dying. High aggregation (or variance) requires collection of more soil samples than lower aggregation to determine mean density (3). In this study, mean density and variance were examined for sampling at different times during the year and with different quadrat sizes. Sampling in the fall for $V$. dahliae required $<10$ samples to determine the mean density within $25 \%$ of the true mean. However, sampling in the spring required 10 to 20 samples, with sample number increasing as fungal density increased, to obtain an estimate within $25 \%$ of the true mean. Fortunately, sampling in the fall provides sufficient time to process samples and implement a management strategy. Time of the year did not change the sample number required for Pratylenchus spp. However, more than 25 samples were required for lesion nematode density near the proposed threshold of 10 vermiforms per $100 \mathrm{~cm}^{3}$ of soil (28). Sample number was reduced to $<10$ as lesion nematode density increased to 100 vermiforms per $100 \mathrm{~cm}^{3}$ of soil. Sample number for M. hapla was high (>20) regardless of time of the year sampled, although required sample number was generally higher (approximately 40 samples at all densities) in the fall than in the spring (20 to 50 samples).

The size of quadrat over which cores were bulked to represent a composite sample did not affect the estimate of $V$. dahliae density and variance until larger $(\geq 1,000$ $\mathrm{m}^{2}$ ) areas were sampled (Table 1 ). It is not unusual in current sampling programs to divide a field into quadrats greater than $4,000 \mathrm{~m}^{2}$. Because of the positive skewness of the negative binomial distribution (3), greatest errors are expected to result in overprediction rather than underprediction of mean densities of $V D$, particularly with the larger quadrat sizes. That is, with small numbers of samples, it is more likely that sample mean will be larger than the true population mean. Quadrat size was not a factor in aggregation of the lesion nematode; the mean density, and to a lesser degree, field site, were more important than quadrat size for this nematode.

The effects of pathogen aggregation can be assessed not only in terms of the altered damage (loss) function (as discussed above), but also in terms of variability (i.e., uncertainty) in predicted yield. Based on

Table 5. Theoretical yields as a function of selected mean densities of Verticillium dahliae (VD), spatial aggregation $(k)$, and presence or absence of Pratylenchus penetrans $(\mathrm{PP})$

\begin{tabular}{lcccccc}
\hline$\overline{V D}$ & $\boldsymbol{k}$ & $\mathbf{P P}$ & $\boldsymbol{v}(\boldsymbol{V D})^{\mathbf{a}}$ & $\mathbf{D E R}^{\mathbf{b}} \times \mathbf{1 0}^{\mathbf{4}}$ & $\boldsymbol{y}(\overline{V D})^{\mathbf{c}}$ & $\overline{\boldsymbol{y}(\boldsymbol{V D})^{\mathbf{d}}}$ \\
\hline 10 & 0.5 & - & 210 & 3.24 & 0.904 & 0.938 \\
10 & 1.5 & - & 77 & 3.24 & 0.904 & 0.916 \\
10 & 3.5 & - & 39 & 3.24 & 0.904 & 0.910 \\
40 & 0.5 & - & 3,240 & 0.73 & 0.765 & 0.883 \\
40 & 1.5 & - & 1,107 & 0.73 & 0.765 & 0.805 \\
40 & 3.5 & - & 497 & 0.73 & 0.765 & 0.783 \\
10 & 0.5 & + & 210 & 5.55 & 0.835 & 0.893 \\
10 & 1.5 & + & 77 & 5.55 & 0.835 & 0.856 \\
10 & 3.5 & + & 39 & 5.55 & 0.835 & 0.845 \\
40 & 0.5 & + & 3,240 & 1.25 & 0.598 & 0.800 \\
40 & 1.5 & + & 1,107 & 1.25 & 0.598 & 0.667 \\
40 & 3.5 & + & 497 & 1.25 & 0.598 & 0.629 \\
\hline
\end{tabular}

${ }^{a}$ The formula for estimating variance of $V D$ is $(\overline{V D})+(\overline{V D})^{2} / k(12)$.

b The second derivative of the yield loss model is: $y^{\prime \prime}(V D)=2\left(1-b_{0}\right) /\left[1,346.89(1+V D / 36.7)^{3}\right]$.

${ }^{\mathrm{c}}$ Yield when spatial aggregation is nonexistent or not considered, $y(V D)=b_{0}+\left(1-b_{0}\right) /[1+$ $\left(V D\right.$ /36.7)], where $b_{0}=0.55$ when PP is absent, and 0.23 when PP is present. $V D$ is mean $V D$.

${ }^{\mathrm{d}}$ Yield when spatial aggregation is considered: $\bar{y}(V D)=y(V D)+y^{\prime \prime}(V D)[v(V D)] / 2$. That is, mean predicted $y$ is a function of mean $V D$ [i.e., $y(V D)$ ] plus a function of the product of the second derivative [i.e., $\left.y^{\prime \prime}(V D)\right]$ evaluated at the mean $(V D)$ and the variation in $V D$ [i.e., $v(V D)$ ]. large-sample theory and the assumption that the parameters are known (not estimated), variance of predicted yield $[v(y)]$ is equal to: $v(V D) \cdot\left[y^{\prime}(\overline{V D})\right]^{2}$, in which $v(V D)$ is the variance of $V D$ (as before), and $y^{\prime}(\overline{V D})$ is the first derivative of $y$ with respect to $V D$, i.e., $y^{\prime}(V D)=-36.7\left(1-b_{0}\right) /(36.7+V D)^{2}$. Thus, choosing a sample size to obtain an acceptably precise estimate of mean $V D$ is the minimum requirement to precisely assess the risks of yield loss in a field. The minimum yield parameter in the yield loss model (29), $b_{0}$, also impacts the variance of predicted yield because $y^{\prime}(\overline{V D})$ is a function of $b_{0}$. Thus, $v(y)$ in the presence of $P$. penetrans will be $(1-0.23)^{2} /(1-0.55)^{2}=$ 2.9 times higher than in the absence of $P$. penetrans (where $b_{0}=0.55$ [29]) when mean and variability of $V$. dahliae microsclerotia are the same. In sampling terms, this means that as the number of sites with both $V$. dahliae and $P$. penetrans increases in a field, more samples will be required to estimate yield losses adequately. This increase in variance of $y$ could be extended to anything that increases the yield loss reduction per unit increase in pathogen density $\left(y^{\prime}\right)$, such as over-irrigation (4) or isolate aggressiveness (1).

Sampling to determine whether to fumigate soils for potato early dying is a common practice in some potato production areas (18). It is important to recognize those factors that impact potato early dying severity and that can be assessed through sampling. Few soilborne fungi can be assessed accurately and consistently in soils, although protocols have been developed for some pathogens (24), including $V$. dahliae (16). Crop managers who base management decisions on soil population density estimates of $V$. dahliae and Pratylenchus spp. could improve their information by maximizing sampling efficiency. This includes collecting sufficient soil samples in the fall after harvest, collecting individual composite samples over relatively small $\left(10\right.$ to $\left.250 \mathrm{~m}^{2}\right)$ randomly selected areas, and increasing sample number appropriately for factors that would increase the potential for damage per unit microsclerotia of $V$. dahliae, such as the presence of $P$. penetrans. A management program could consist of soil sampling in the fall, with two to seven samples per field, depending on damage threshold (a damage threshold near $10 \mathrm{CFU} / \mathrm{cm}^{3}$ of soil would require seven samples, while one near $100 \mathrm{CFU} / \mathrm{cm}^{3}$ of soil would require two soil samples). The number of samples would increase: if $P$. penetrans was present (by 2.9 times), if the isolate of $V$. dahliae in a particular field was unusually aggressive, or if planning for the most diseaseconducive weather. It is essential to estimate or have records for the lowest possible minimum yield experienced in that field due to potato early dying. That value can be substituted into the yield loss model, and the number of extra samples 
necessary for a given scenario can be calculated. The most difficult part of selecting a minimum sampling number is to know how much loss to attribute to potato early dying.

\section{ACKNOWLEDGMENTS}

Salaries and research support were provided by state and federal funds appropriated to the Ohio Agricultural Research and Development Center, The Ohio State University, and grants from the USDA/NCIPM. Comments provided by our internal reviewers, Tom Archer, TAES-Lubbock, and Linda Allen of Texas Tech University, were most helpful in writing this manuscript.

\section{LITERATURE CITED}

1. Botseas, D. D., and Rowe, R. C. 1994. Development of potato early dying in response to infection by two pathotypes of Verticillium dahliae and co-infection by Pratylenchus penetrans. Phytopathology 84:275-282.

2. Bowers, J. H., Nameth, S. T., Riedel, R. M., and Rowe, R. C. 1996. Infection and colonization of potato roots by Verticillium dahliae as affected by Pratylenchus penetrans and $P$. crenatus. Phytopathology 86:614-621.

3. Campbell, C. L., and Madden, L. V. 1990. Introduction to Plant Disease Epidemiology. John Wiley \& Sons, New York.

4. Cappaert, M. R., Powelson, M. L., Christensen, N. W., Stevenson, W. R., and Rouse, D. I. 1994. Assessment of irrigation as a method of managing potato early dying. Phytopathology 84:792-800.

5. Evans, G., and Gleeson, A. C. 1980. An evaluation of the sampling variation when estimating the population of Verticillium dahliae in field soil. Ann. Appl. Biol. 95:177-184.

6. Ferrandino, F. J. 1989. A distribution-free method for estimating the effect of aggregated plant damage on crop yield. Phytopathology 79:1229-1232.

7. Francl, L. J., and Wheeler, T. A. 1993. Interaction of plant parasitic nematodes with wiltinducing fungi. Pages 79-103 in: Nematode Interactions. M. W. Khan, ed. Chapman \& Hall, London, England.
8. Handoo, Z. A., and Golden, A. M. 1989. A key and diagnostic compendium to the species of the genus Pratylenchus Filipjev, 1936 (lesion nematodes). J. Nematol. 21:202-218.

9. Hughes, G. 1990. Characterizing crop responses to patchy pathogen attack. Plant $\mathrm{Pa}-$ thol. 39:2-4.

10. Jacobsen, B. J., MacDonald, D. H., and Bissonnette, H. L. 1979. Interaction between Meloidogyne hapla and Verticillium alboatrum in the Verticillium wilt disease of potato. Phytopathology 69:288-292.

11. Johnson, K. B., Apple, J. D., and Powelson, M. L. 1988. Spatial patterns of Verticillium dahliae propagules in potato field soils of Oregon's Columbia Basin. Plant Dis. 72:484488.

12. MacGuidwin, A. E., and Rouse, D. I. 1990. Role of Pratylenchus penetrans in the potato early dying disease of Russet Burbank potato. Phytopathology 80:1077-1082.

13. Madden, L. V., and Nutter, F. W., Jr. 1995. Modeling crop losses at the field scale. Can. J. Plant Pathol. 17:124-137.

14. Martin, M. J., Riedel, R. M., and Rowe, R. C. 1982. Verticillium dahliae and Pratylenchus penetrans: Interactions in the early dying complex of potato in Ohio. Phytopathology 72:640-644.

15. Milliken, G. A., and Johnson, D. E. 1984. Analysis of Messy Data. Vol. I. Designed Experiments. Lifetime Learning Publications, Belmont, CA.

16. Nicot, P. C., and Rouse, D. I. 1987. Precision and bias of three quantitative soil assays for Verticillium dahliae. Phytopathology 77:875881.

17. Noe, J. P., and Barker, K. R. 1985. Overestimation of yield loss of tobacco caused by the aggregated spatial pattern of Meloidogyne incognita. J. Nematol. 17:245-251.

18. Powelson, M. L., and Rowe, R. C. 1993. Biology and management of early dying of potatoes. Annu. Rev. Phytopathol. 31:111126.

19. Pullman, G. S., and DeVay, J. E. 1982. Epidemiology of Verticillium wilt of cotton: A relationship between inoculum density and disease progression. Phytopathology 72:549-
554.

20. Rowe, R. C., Davis, J. R., Powelson, M. L., and Rouse, D. I. 1987. Potato early dying: Causal agents and management strategies. Plant Dis. 71:482-489.

21. Saeed, I. A. M., MacGuidwin, A. E., and Rouse, D. I. 1998. Effect of initial nematode population density on the interaction of Pratylenchus penetrans and Verticillium dahliae on 'Russet Burbank' potato. J. Nematol. 30:100-107.

22. Schreiber, L. R., and Green, R. J. 1962. Comparative survival of mycelium, conidia, and microsclerotia of Verticillium albo-atrum in mineral soil. Phytopathology 52:288-289.

23. Seinhorst, J. W. 1973. The relation between nematode distribution in a field and loss in yield at different average nematode densities. Nematologica 19:421-427.

24. Singleton, L. L., Mihail, J. D., and Rush, C. M., eds. 1993. Methods for Research on Soilborne Phytopathogenic Fungi. American Phytopathological Society, St. Paul, MN.

25. Smith, V. L., and Rowe, R. C. 1984. Characteristics and distribution of propagules of Verticillium dahliae in Ohio potato field soils and assessment of two assay methods. Phytopathology 74:553-556.

26. Taylor, L. R. 1961. Aggregation, variance and the mean. Nature 189:732-735

27. Thistlethwayte, B. 1970. Reproduction of Pratylenchus penetrans (Nematoda: Tylenchida). J. Nematol. 2:101-105.

28. Wheeler, T. A., Madden, L. V., Riedel, R. M., and Rowe, R. C. 1994. Distribution and yield-loss relations of Verticillium dahliae, Pratylenchus penetrans, $P$. scribneri, $P$ crenatus, and Meloidogyne hapla in commercial potato fields. Phytopathology 84:843-852.

29. Wheeler, T. A., Madden, L. V., Rowe, R. C., and Riedel, R. M. 1992. Modeling of yield loss in potato early dying caused by Pratylenchus penetrans and Verticillium dahliae. $\mathrm{J}$ Nematol. 24:99-102.

30. Wheeler, T. A., and Rowe, R. C. 1995. Influence of soil characteristics and assay techniques on quantification of Verticillium dahl iae in Ohio soils. Plant Dis. 79:29-34. 